NASA/TM-2012-217699

\title{
Optimization of Low-Thrust Spiral Trajectories by Collocation
}

Robert D. Falck

Glenn Research Center, Cleveland, Ohio

John W. Dankanich

AeroDank, Inc., Cleveland, Ohio 


\section{NASA STI Program . . . in Profile}

Since its founding, NASA has been dedicated to the advancement of aeronautics and space science. The NASA Scientific and Technical Information (STI) program plays a key part in helping NASA maintain this important role.

The NASA STI Program operates under the auspices of the Agency Chief Information Officer. It collects, organizes, provides for archiving, and disseminates NASA's STI. The NASA STI program provides access to the NASA Aeronautics and Space Database and its public interface, the NASA Technical Reports Server, thus providing one of the largest collections of aeronautical and space science STI in the world. Results are published in both non-NASA channels and by NASA in the NASA STI Report Series, which includes the following report types:

- TECHNICAL PUBLICATION. Reports of completed research or a major significant phase of research that present the results of NASA programs and include extensive data or theoretical analysis. Includes compilations of significant scientific and technical data and information deemed to be of continuing reference value. NASA counterpart of peer-reviewed formal professional papers but has less stringent limitations on manuscript length and extent of graphic presentations.

- TECHNICAL MEMORANDUM. Scientific and technical findings that are preliminary or of specialized interest, e.g., quick release reports, working papers, and bibliographies that contain minimal annotation. Does not contain extensive analysis.

- CONTRACTOR REPORT. Scientific and technical findings by NASA-sponsored contractors and grantees.
- CONFERENCE PUBLICATION. Collected papers from scientific and technical conferences, symposia, seminars, or other meetings sponsored or cosponsored by NASA.

- SPECIAL PUBLICATION. Scientific, technical, or historical information from NASA programs, projects, and missions, often concerned with subjects having substantial public interest.

- TECHNICAL TRANSLATION. Englishlanguage translations of foreign scientific and technical material pertinent to NASA's mission.

Specialized services also include creating custom thesauri, building customized databases, organizing and publishing research results.

For more information about the NASA STI program, see the following:

- Access the NASA STI program home page at http://www.sti.nasa.gov

- E-mail your question to help@sti.nasa.gov

- Fax your question to the NASA STI Information Desk at 443-757-5803

- Phone the NASA STI Information Desk at 443-757-5802

- Write to: STI Information Desk NASA Center for AeroSpace Information 7115 Standard Drive Hanover, MD 21076-1320 
NASA/TM-2012-217699

AIAA-2012-4423

\section{Optimization of Low-Thrust Spiral Trajectories by Collocation}

Robert D. Falck

Glenn Research Center, Cleveland, Ohio

John W. Dankanich

AeroDank, Inc., Cleveland, Ohio

Prepared for the

2012 Astrodynamics Specialists Conference

sponsored by the American Institute of Aeronautics and Astronautics (AIAA) and American Astronautical Society (AAS)

Minneapolis, Minnesota, August 13-16, 2012

National Aeronautics and

Space Administration

Glenn Research Center

Cleveland, Ohio 44135 


\section{Acknowledgments}

The work described in this paper was funded in whole or in part by the In-Space Propulsion Technology Program, which is managed by NASA's Science Mission Directorate in Washington, D.C., and implemented by the In-Space Propulsion Technology Project at the NASA Glenn Research Center in Cleveland, Ohio.

This report is a formal draft or working paper, intended to solicit comments and ideas from a technical peer group. subject to revision as analysis proceeds.

Trade names and trademarks are used in this report for identification only. Their usage does not constitute an official endorsement, either expressed or implied, by the National Aeronautics and Space Administration.

Level of Review: This material has been technically reviewed by technical management.

Available from

NASA Center for Aerospace Information 7115 Standard Drive

Hanover, MD 21076-1320
National Technical Information Service 5301 Shawnee Road Alexandria, VA 22312

Available electronically at http://www.sti.nasa.gov 


\title{
Optimization of Low-Thrust Spiral Trajectories by Collocation
}

\author{
Robert D. Falck \\ National Aeronautics and Space Administration \\ Glenn Research Center \\ Cleveland, Ohio 44135 \\ John W. Dankanich \\ AeroDank, Inc. \\ Cleveland, Ohio 44135
}

\begin{abstract}
As NASA examines potential missions in the post space shuttle era, there has been a renewed interest in low-thrust electric propulsion for both crewed and uncrewed missions. While much progress has been made in the field of software for the optimization of low-thrust trajectories, many of the tools utilize higher-fidelity methods which, while excellent, result in extremely high run-times and/or poor convergence when dealing with planetocentric spiraling trajectories deep within a gravity well. Conversely, faster tools like SEPSPOT provide a reasonable solution but typically fail to account for other forces such as third-body gravitation, aerodynamic drag, solar radiation pressure. SEPSPOT is further constrained by its solution method, which may require a very good guess to yield a converged optimal solution. Here the authors have developed an approach using collocation intended to provide solution times comparable to those given by SEPSPOT while allowing for greater robustness and extensible force models.
\end{abstract}

\section{Nomenclature}

Time, days

State variable vector

Time-varying control variable vector

Global design variable vector

Phase design variable vector

Initial boundary constraint

Final boundary constraint

Path constraint

Non-dimensional time within a segment polynomial

Continuity defect

Differential defect

Lagrange interpolation matrix for interior nodes

Lagrange derivative matrix for interior nodes

Lagrange derivative matrix for cardinal nodes

Number of state variables

Number of time-varying control variables

Number of cardinal nodes per phase

Number of interior nodes per phase

Independent variable vector in the nonlinear optimization problem

Boundary constraint vector for the nonlinear optimization problem

Semi-major axis, km

Eccentricity

Inclination, deg 


\begin{tabular}{|c|c|}
\hline$\Omega$ & Right ascension of ascending node, deg \\
\hline$\omega$ & Argument of periapsis, deg \\
\hline$\theta$ & True anomaly, deg \\
\hline$h$ & Specific angular momentum, $\frac{\mathrm{km}^{2}}{\mathrm{sec}}$ \\
\hline$p$ & Semilatus rectum, $\mathrm{km}$ \\
\hline$f$ & $\mathrm{X}$-component of eccentricity vector in the equinoctial frame \\
\hline$g$ & Y-component of eccentricity vector in the equinoctial frame \\
\hline$j$ & $\mathrm{X}$-component of ascending node vector in the equinoctial frame \\
\hline$k$ & Y-component of ascending node vector in the equinoctial frame \\
\hline$L$ & True longitude, deg \\
\hline$r$ & Spacecraft radius from central body, $\mathrm{km}$ \\
\hline$v$ & Spacecraft velocity in the EME2000 frame, $\frac{\mathrm{km}}{\mathrm{sec}}$ \\
\hline$m$ & Spacecraft mass, $\mathrm{kg}$ \\
\hline rev & Number of orbital revolutions \\
\hline$T_{p}$ & Orbital period, days \\
\hline$P Q W$ & Perifocal coordinate frame \\
\hline$N T W$ & Velocity-vector aligned spacecraft coordinate frame \\
\hline$R S W$ & Local-vertical, local-horizontal spacecraft coordinate frame \\
\hline$E M E 2000$ & Earth Mean Equator of J2000 inertial coordinate frame \\
\hline$r_{c b}$ & Central body equatorial radius, $\mathrm{km}$ \\
\hline$\mu$ & Gravitational parameter of the central body, $\frac{\mathrm{km}^{3}}{\mathrm{~s}^{2}}$ \\
\hline$P_{0}$ & Spacecraft propulsion system input power, kW \\
\hline$\eta$ & Spacecraft propulsion system efficiency \\
\hline$I_{s p}$ & Spacecraft propulsion system specific impulse, sec \\
\hline Subscript & \\
\hline$L$ & Lower bound \\
\hline$U$ & Upper bound \\
\hline$i$ & Interior node \\
\hline$c$ & Cardinal node \\
\hline 0 & Initial condition \\
\hline$f$ & Final condition \\
\hline
\end{tabular}

\section{Introduction}

GOR several decades, SEPSPOT has been NASA's primary tool for the optimization of planetocentric $\mathrm{H}$ low-thrust trajectories. ${ }^{1}$ SEPSPOT takes advantage of orbital-averaging techniques to greatly increase the speed of computation at the expense of fidelity. Generally, orbital averaging techniques provide an accurate answer regarding the transfer of one orbit to another, but lack the precision to put a spacecraft at a given position at a specific time. Although relatively fast, SEPSPOT suffers from some weaknesses that result in difficulty when using it to analyze state-of-the-art low-thrust transfers. SEPSPOT utilizes indirect optimization techniques based on Hamilton-Lagrange theory. In addition to providing an initial guess at values of the orbital state variables, one must also provide a fairly accurate guess for initial and final values of the costates, which can be challenging. More critically, extending the equations of motion to include additional force models (atmospheric drag, solar pressure, perturbations by the Moon and Sun, etc.) would require the equations of motion of the costate variables to be rederived from their current form.

To address these issues, the authors have embarked on developing an approach which uses a collocation technique to transform an initial-value-problem (IVP) or two-point boundary value problem (TPBVP) into a nonlinear programming (NLP) problem, which may be solved utilizing an off-the-shelf optimization software package. Since this approach utilizes direct optimization method, it has the advantage that new environmental and vehicle related force models may be added with relative ease. Collocation and pseudospectral optimization techniques were demonstrated by Dickmanns and Well ${ }^{2}$ and Hargraves and Paris ${ }^{3}$ and have been used with success in software such as OTIS $4,{ }^{4}$ DIDO,${ }^{5}$ and SOCS. ${ }^{6}$ Collocation and pseudospectral methods have previously been applied to the low-thrust spiraling problem by Betts. ${ }^{7}$ However, in his work he directly transcribed the equinoctial equations of motion with respect to time, which led to a mesh grid 
of over 16000 points and a run time of several hours. ${ }^{7}$ Rather than take this approach, which sees highly oscillatory behavior in the time-histories of the states and controls, the authors utilize orbital averaging techniques in conjunction with hybrid control formulations which largely remove the oscillatory behavior from the problem. This substantially reduces the number and order of the collocating polynomials, which reduces the size of the NLP problem and results in run times on the order of a few seconds to minutes.

Kluever demonstrated an orbital averaging problem which utilizes the Gauss form of the Lagrange planetary equations in conjunction with a hybrid control technique. ${ }^{8}$ Using only a handful of mesh points for the controls, he demonstrated the ability to optimize a LEO to GEO transfer in good agreement with SEPSPOT. For this approach the authors elected to use collocation of the states and controls since the method tends to show an ability to find optimal solutions given a relatively poor initial guess. The authors use equations of motion based on a modified set of equinoctial elements, which don't suffer from as many singularities as the Lagrange planetary equations.

Like Kluever, the authors utilize a hybrid set of control variables. These control variables are the costates of the Hamilton-Lagrange formulation. They show a relatively smooth time-history throughout an orbital transfer, which is beneficial for collocation, but internally are transformed into a set of pitch and yaw angles which may oscillate greatly over the course of any single orbit. Furthermore, due to the extensible nature of the collocated form of this problem, alternative guidance strategies can be easily implemented so long as they can be parameterized such that their control variables do not exhibit "excessively" oscillatory behavior throughout the orbital transfer. As such, this method allows users to determine both the optimal (minimum time, minimum propellant, etc.) transfer guidance history, but also how it compares to a custom guidance strategy. The use of collocation to solve the optimal control problem should, in general, result in a greater radius of convergence such that the initial guesses to the costates need not be as accurate as those supplied to SEPSPOT.

This approach does sacrifice fidelity for speed, and thus is intended for the preliminary analysis of lowthrust trajectories and guidance algorithm development. For example, orbital averaging is incapable of analyzing a lunar insertion maneuver as demonstrated by Betts. However, the run-time is so small that using a collocated averaging technique to "dig" out of the deepest part of the gravity well would greatly reduce analysis time. Once at a sufficiently high intermediate orbit, the oscillatory behavior of the state and control time histories is low enough to permit collocation of non-averaged equations of motion from the intermediate orbit to the final target.

\section{Formulation}

\section{A. The Collocation Problem}

Collocation is used to simultaneously simulate and optimize the trajectory of a dynamical system. As a fallback, the implementation also supports a more traditional explicitly integrated shooting method for trajectory optimization, though experience has shown that implicit integration is not only faster, but often capable of finding more optimal solutions.

In general terms, the optimization problem is of the following form:

$$
\begin{array}{ll}
\text { Minimize } & f_{o b j}\left(\mathbf{x}\left(\mathbf{t}_{\mathbf{0} \mid \mathbf{f}}\right), t_{0 \mid f}, \mathbf{u}\left(\mathbf{t}_{\mathbf{0} \mid \mathbf{f}}\right), \phi_{\mathbf{g}}, \phi_{\mathbf{p}}\right) \\
\text { s.t. } & \mathbf{x}_{\mathbf{L}} \leq \mathbf{x} \leq \mathbf{x}_{\mathbf{U}} \\
& t_{L} \leq t \leq t_{U} \\
& \mathbf{u}_{\mathbf{L}} \leq \mathbf{u} \leq \mathbf{u}_{\mathbf{U}} \\
& \phi_{\mathbf{g}, \mathbf{L}} \leq \phi_{\mathbf{g}} \leq \phi_{\mathbf{g}, \mathbf{U}} \\
& \phi_{\mathbf{p}, \mathbf{L}} \leq \phi_{\mathbf{p}} \leq \phi_{\mathbf{p}, \mathbf{U}} \\
& \psi_{\mathbf{0}, \mathbf{L}} \leq f_{\psi_{0}}\left(\mathbf{x}\left(\mathbf{t}_{\mathbf{0}}\right), t_{0}, \mathbf{u}\left(\mathbf{t}_{\mathbf{0}}\right), \phi_{\mathbf{g}}, \phi_{\mathbf{p}}\right) \leq \psi_{\mathbf{0}, \mathbf{U}} \\
& \psi_{\mathbf{f}, \mathbf{L}} \leq f_{\psi_{0}}\left(\mathbf{x}\left(\mathbf{t}_{\mathbf{f}}\right), t_{f}, \mathbf{u}\left(\mathbf{t}_{\mathbf{f}}\right), \phi_{\mathbf{g}}, \phi_{\mathbf{p}}\right) \leq \psi_{\mathbf{f}, \mathbf{U}} \\
& \psi_{\mathbf{p}, \mathbf{L}} \leq f_{\text {path }}\left(\mathbf{x}(\mathbf{t}), t, \mathbf{u}(\mathbf{t}), \phi_{\mathbf{g}}, \phi_{\mathbf{p}}\right) \leq \psi_{\mathbf{p}, \mathbf{U}}
\end{array}
$$

Where $\mathbf{x}$ and $\mathbf{u}$ are the time-varying states and controls, respectively. The vectors $\phi_{\mathbf{g}}$ and $\phi_{\mathbf{p}}$ contain static optimization variables (design variables) for the entire problem, and a specific phase, respectively. 
Variable $t$ is the time at the point of the objective function evaluation; either the beginning or end of a phase. All states, controls, design variables, and time may have simple bounds. Furthermore, we may impose boundary constraints at the start or end of a phase $\left(\psi_{0}\right.$ and $\left.\psi_{f}\right)$, or path constraints to be assessed throughout a phase $\left(\psi_{p}\right)$.

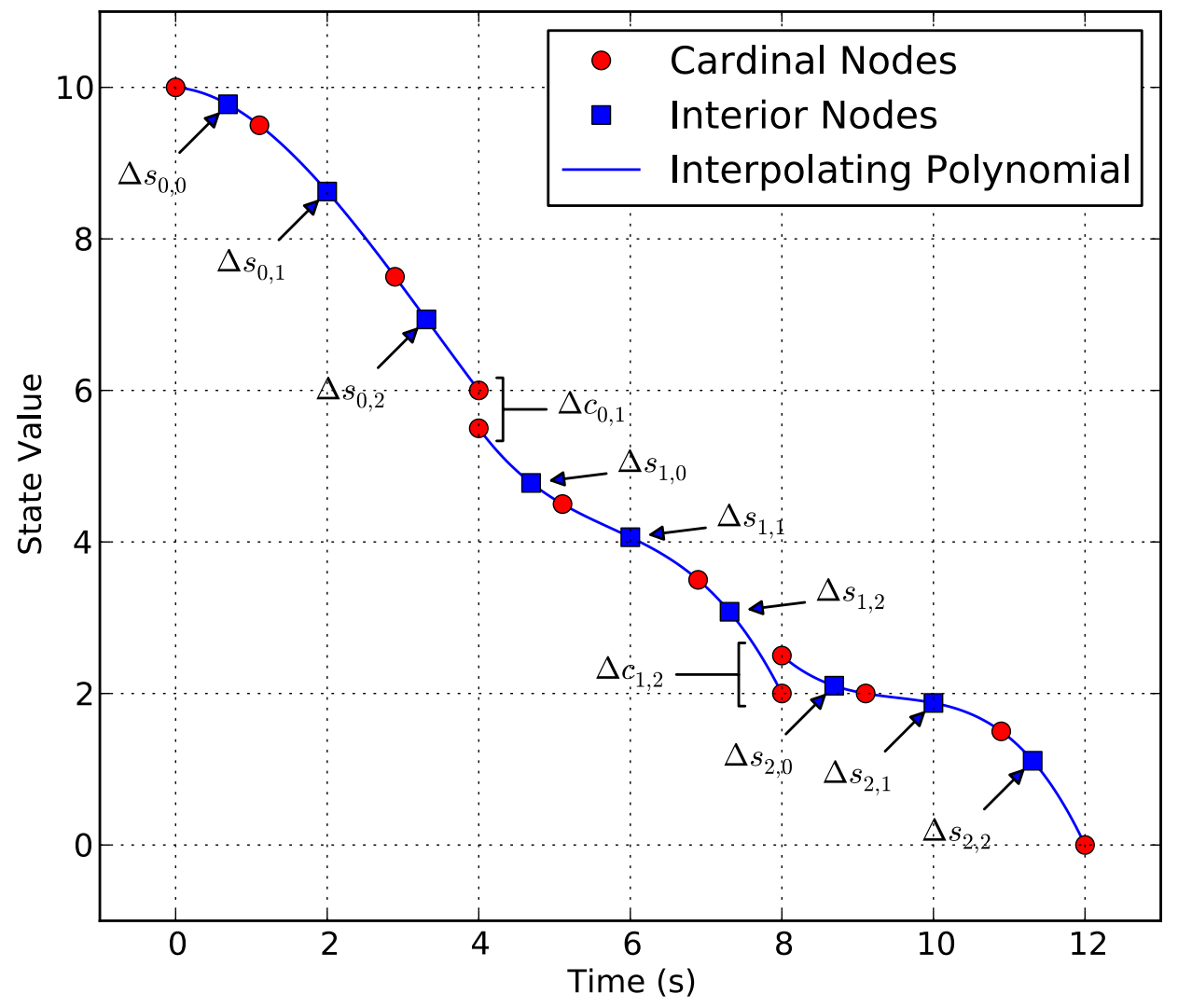

Figure 1. The state time-history of a single state in a phase consisting of three 3rd-order segments, including the differential defects $\left(\Delta s_{i, j}\right)$ and continuity defects $\left(\Delta c_{i, j}\right)$

The collocation problem consists of a series of phases in which the forces acting upon the spacecraft are consistent such that the states are $C^{1}$ continuous within a phase. Each phase is defined on an interval $\left[t_{0}, t_{0}+t_{p}\right]$, and the equations of motion dictate some set of states $\mathbf{x}$ and controls $\mathbf{u}$. Each phase is further subdivided into a series of segments along which the states and controls are represented by a polynomial of at least order 3 , and thus $C^{2}$ continuous. For each state and control along each segment, this polynomial is defined at a series of nodes called the cardinal nodes. Internally, each segment of order $o$ is defined on the interval $[-1,1]$ (so called $\tau$-space) and has cardinal nodes at the $(o+1)$ Legendre-Gauss-Lobatto points. For example, a 3rd-order polynomial segment has four cardinal nodes. The collocation engine uses a nonlinear optimization routine to vary the cardinal values of the states and controls, the phase start time and duration, and design variable values such that state time histories accurately reflect the equations of motion and the objective function (1) is satisfied.

The accuracy of the collocated polynomials in representing the dynamics of the system is measured by comparing the slope of the polynomial for a given state to the derivative of that state as given by the equations of motion. These differences are called differential defects. A well-posed initial-value-problem has a unique solution and requires that the number of independent variables is equal to the number of equality constraints. Figure 1 illustrates the setup of a collocation problem for one state variable in a phase of three 3rd-order polynomial segments. With just one state this problem has 14 variables: the initial time and phase duration (2), and the state values at four cardinal nodes in each of three segments (12). We require that 
there be no discontinuity in the value of a state variable at the segment boundaries.

$$
\Delta \mathrm{c}_{\mathrm{i}, \mathrm{j}}=\mathrm{x}_{\mathrm{c}, \mathrm{j}}-\mathrm{x}_{\mathrm{c}, \mathrm{i}}=\mathbf{0}
$$

Furthermore, the initial time, duration, and initial state value in this phase are fixed via bounds, giving a total of five equality constraints. The remaining nine equality constraints are achieved by requiring the differential defects at three points in each segment to be zero. The points in each polynomial where the defects are assessed are referred to as the interior nodes. Within each segment we may construct a Lagrange interpolation matrix $\mathbf{L}_{\mathbf{i}}$ and a Lagrange derivative matrix $\mathbf{D}_{\mathbf{i}}$ such that the values and derivatives of the states and controls at the interior nodes may be obtained by simple matrix-vector products:

$$
\begin{aligned}
\mathbf{x}_{\mathbf{i}} & =\mathbf{L}_{\mathbf{i}} \mathbf{x}_{\mathbf{c}} \\
\frac{d \mathbf{x}_{\mathbf{i}}}{d t} & =\mathbf{D}_{\mathbf{i}} \mathbf{x}_{\mathbf{c}} \frac{d \tau}{d t}
\end{aligned}
$$

Finally, the differential defects of the states at the interior node of the phase are:

$$
\boldsymbol{\Delta}_{\mathbf{i}}=f_{\text {eom }}\left(\mathbf{x}_{\mathbf{i}}, t_{i}, \mathbf{u}_{\mathbf{i}}, \phi_{\mathbf{g}}, \phi_{\mathbf{p}}\right)-\frac{d \mathbf{x}_{\mathbf{i}}}{d t}=\mathbf{0}
$$

Some preliminary tests suggest that a good choice of interior nodes is to determine the LGL points for an $(o+1)$ polynomial and remove the endpoints. Reusing the first (or last) $o$ cardinal nodes as the interior nodes is also possible, but generally exhibits poorer convergence. On the other hand, if the convergence issues can be fixed, reusing the cardinal nodes as interior nodes would reduce equation of motion evaluations by nearly a factor of two, and no matrix multiplications (11) would be required to determine the state and control values at the interior nodes.

Experience has also shown that convergence is aided by requiring both value and rate continuity to be imposed on the control values at the segment boundaries. Control rates at the segment boundaries are determined with a Lagrange derivative matrix constructed such that it returns the derivatives of the polynomial at the cardinal nodes:

$$
\frac{d \mathbf{u}_{\mathbf{c}}}{d t}=\mathbf{D}_{\mathbf{c}} \mathbf{u}_{\mathbf{c}} \frac{d \tau}{d t}
$$

\section{B. The NLP Interface}

The optimization problem above is transcribed by the collocation engine into the following form:

$$
\begin{array}{ll}
\text { Minimize } & f_{\text {obj }}(\mathbf{z}) \\
\text { s.t. } & \mathbf{g}_{\mathbf{L}} \leq \mathbf{g}(\mathbf{z}) \leq \mathbf{g}_{\mathbf{U}} \\
& \mathbf{z}_{\mathbf{L}} \leq \mathbf{z} \leq \mathbf{z}_{\mathbf{U}}
\end{array}
$$

This transformed optimization problem may be solved by a variety of off-the-shelf nonlinear optimization software packages. Currently the authors are using the IPOPT ${ }^{9}$ which is able to capitalize on the sparsity of the Jacobian matrix that is characteristic of the collocaiton problem.

The times, states, controls, and design parameters are scaled and packed into the independent variable array for the NLP $(\mathbf{z})$. Currently a basic scaling scheme is used whereby each independent variable is scaled by the inverse of its expected magnitude such that the corresponding component of $\mathbf{z}$ is roughly on the order of one. The independent parameter array is packed in the following order:

1. The scaled global design parameter values comprise the first elements of $\mathbf{z}$

2. The scaled design parameter values of the first phase comprise the next elements of $\mathbf{z}$ 
3. The scaled initial time and duration of the first phase are the next two elements of $\mathbf{z}$

4. The cardinal values of the $n$ states and $m$ controls of the first phase are packed as the next $(n+m) \cdot n c n_{p}$ elements, where $n c n_{p}$ is the number of cardinal nodes in the phase. These values are packed such that the states and columns at any given cardinal node are contiguous. Conceptually, this is the same as constructing the following $n c n_{p}$ by $n+m$ matrix $\mathbf{X}_{\mathbf{c}} \mathbf{U}_{\mathbf{c}}$ and unraveling its values in row-major order.

$$
\mathbf{X}_{\mathbf{c}} \mathbf{U}_{\mathbf{c}}=\left[\begin{array}{ccccccc}
x_{0,0} & x_{1,0} & \cdots & x_{n-1,0} & u_{0,0} & \cdots & u_{m-1,0} \\
x_{0,1} & x_{1,1} & \cdots & x_{n-1,1} & u_{0,1} & \cdots & u_{m-1,1} \\
\vdots & \vdots & \ddots & \vdots & \vdots & \vdots & \vdots \\
x_{0, n c n_{p}-1} & x_{1, n c n_{p}-1} & \cdots & x_{n-1, n c n_{p}-1} & u_{0, n c n_{p}-1} & \cdots & u_{m-1, n c n_{p}-1}
\end{array}\right]
$$

5. Items 2 through 4 are repeated for each phase in the problem.

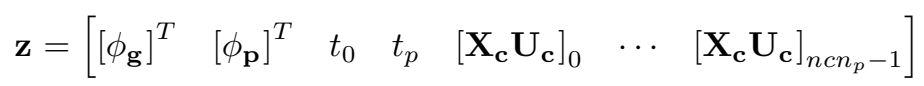

Similarly, the constraints of the collocation problem are packed into the NLP constraint array (g) array in the following order:

1. The phase linkage constraints comprise the first elements of $\mathbf{g}$

2. The $\operatorname{nin}_{p} \cdot n$ state defects for the first phase are stored node-by-node as the next elements of $\mathbf{g}$.

3. The state and control continuity conditions at each of the num_seg -1 segment boundaries in the first phase make up the next (num_seg -1$) \cdot(n+m)$ elements of $\mathbf{g}$.

4. The control continuity rate conditions at each of the num_seg-1 segment boundaries in the first phase make up the next $($ num_seg-1) $\cdot m$ elements of $\mathbf{g}$.

5. The initial and final boundary constraints of the first phase make up the next elements of $\mathbf{g}$

6. Finally, the path constraints are evaluated at each cardinal node and comprise the final $n c n_{p} \cdot n p c$ elements of $\mathbf{g}$, where ncp is the number of path constraints in the phase.

7. Items 2 through 6 are repeated for each phase in the problem.

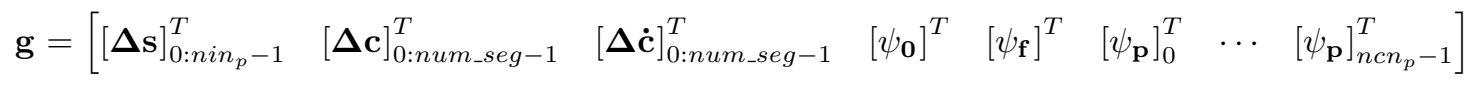

By ordering the $\mathbf{z}$ and $\mathbf{g}$ arrays in such a way the sparsity pattern of the Jacobian matrix is largely block-diagonal. The use of a nonlinear programming routine which is able to capitalize on the sparsity of the Jacobian matrix is a critical feature which enables the solution to the collocation problem to be obtained rapidly.

\section{Equations of Motion}

In his work, Kluever showed that, by applying orbital averaging to the Gauss form of the Lagrange planetary equations, ${ }^{10}$ he was able to get very good agreement with SEPSPOT results by parameterizing the controls at some set of control nodes and explicitly integrating the solution. ${ }^{8}$ However, the Lagrange planetary equations are problematic in the context of a collocation formulation since they exhibit singularities at zero eccentricity and zero inclination, a point which is of interest for the purpose of examining trajectories to or from geostationary orbit. Instead, the authors utilized a set of modified equinoctial elements as given by Walker. ${ }^{11}$ Betts also utilized this set of equations of motion in his low-thrust orbit transfer example, though he did not employ an averaging technique. ${ }^{12}$ 


$$
\dot{x}=\left[\begin{array}{ccc}
0 & \frac{2 p}{w} \sqrt{\frac{p}{\mu}} & 0 \\
\sqrt{\frac{p}{\mu}} \sin (L) & \sqrt{\frac{p}{\mu}} \frac{[(w+1) \cos (L)+f]}{w} & -\sqrt{\frac{p}{\mu}} \frac{g(j \sin (L)-k \cos (L))}{w} \\
-\sqrt{\frac{p}{\mu}} \cos (L) & \sqrt{\frac{p}{\mu} \frac{[(w+1) \sin (L)+g]}{w}} & \sqrt{\frac{p}{\mu}} \frac{f(j \sin (L)-k \cos (L))}{w} \\
0 & 0 & \sqrt{\frac{p}{\mu}} \frac{s^{2}}{2 w} \cos (L) \\
0 & 0 & \sqrt{\frac{p}{\mu} \frac{s^{2}}{2 w} \sin (L)} \\
0 & 0 & \sqrt{\frac{p}{\mu}} \frac{j \sin (L)-k \cos (L)}{w} \\
0 & 0 & 0 \\
0 & 0 & 0
\end{array}\right]\left[\begin{array}{c}
a_{r} \\
a_{s} \\
a_{w}
\end{array}\right]+\left[\begin{array}{c}
0 \\
0 \\
0 \\
0 \\
0 \\
\sqrt{\mu p}\left(\frac{q}{p}\right)^{2} \\
-\dot{m} \\
r \dot{e} v
\end{array}\right]
$$

where the state vector consists of the modified equinoctial elements and spacecraft mass

$$
\mathbf{x}=\left[\begin{array}{llllllll}
p & f & g & j & k & L & m & r e v
\end{array}\right]^{T}
$$

the accelerations

$$
\mathbf{a}=\left[\begin{array}{c}
a_{r} \\
a_{s} \\
a_{w}
\end{array}\right]
$$

are the radial, local-horizontal, and orbit normal perturbing accelerations where

$$
\begin{aligned}
s^{2} & =1+j^{2}+k^{2} \\
w & =\frac{p}{r}=1+f \cos (L)+g \sin (L)
\end{aligned}
$$

Note we refer to the equinoctial element $h$ as $j$ to avoid confusion with the specific angular momentum of the orbit. The equations of motion (22) account for central-body gravitation in the absence of perturbing accelerations. Adding different perturbing accelerations is as simple as converting them to the RSW frame and including them in (24). For the purposes of this paper, the only non-two-body accelerations are due to thrust and $J_{2}$ perturbations.

The mass flow rate $(\dot{m})$ is based on the propulsion system model (29).

The number of orbital revolutions performed by the spacecraft can be approximated by treating it as an integrated state variable with a derivative function equal to the frequency of the spacecraft orbit.

$$
r \dot{e} v=\frac{1}{T_{p}}=\frac{\sqrt{\mu}}{2 \pi a^{\frac{3}{2}}}
$$

\section{Solar Electric Propulsion Model}

The solar electric propulsion system generates power based on its distance to the Sun and the angle between the array plane and the Sun vector. In the analysis below the Sun is assumed to be 1 A.U. from the spacecraft at all times and the arrays are always perfectly pointed at the Sun. If the nominal array power at 1 A.U. is given by $P_{0}$, then the spacecraft acceleration due to thrust is ${ }^{8}$

$$
a_{T}=\frac{2 \eta P_{0}}{m g I_{s p}}
$$

and the rate of propellant expenditure due to engine firing is derived from the definition of thrust:

$$
F_{T}=\dot{m} g I_{s p} \longrightarrow \dot{m}=\frac{2 \eta P_{0}}{g^{2} I_{s p}^{2}}
$$




\section{E. Gravitational Perturbations due to Oblateness of the Central Body}

Kechichian quantified $J_{2}$ perturbations in the RSW frame as: ${ }^{13}$

$$
\mathbf{a}_{\mathbf{J} 2}=\left[\begin{array}{c}
-\frac{3 \mu J_{2} r_{c b}^{2}}{2 r^{4}}\left(1-12 \frac{(j \sin (L)-k \cos (L))^{2}}{s^{4}}\right) \\
-\frac{12 \mu J_{2} r_{c b}^{2}}{r^{4}} \frac{(j \sin (L)-k \cos (L))(j \cos (L)+k \sin (L))}{s^{4}} \\
-\frac{6 \mu J_{2} r_{c b}^{2}}{r^{4}} \frac{(j \sin (L)-k \cos (L))\left(1-k^{2}-j^{2}\right)}{s^{4}}
\end{array}\right]
$$

\section{F. Orbital Averaging}

The equations of motion (22) are subject to oscillatory behavior which can greatly increase the number of collocation segments required for a converged solution. To enable better performance from the collocation routine, the equations of motion are averaged. The averaged equations of motion are obtained by converting $\dot{\mathbf{x}}$ to $\frac{d \mathbf{x}}{d \theta}$, integrating for an entire orbital revolution, and dividing by the orbital period to obtain the average rates of change in the modified equinoctial elements for a given orbital state. ${ }^{8}$ The anomaly term (true longitude for the modified equinoctial elements) is not included among the averaged orbital elements.

$$
\overline{\dot{\mathbf{x}}}=\frac{1}{T_{p}} \int_{-\pi}^{\pi} \frac{d \mathbf{x}}{d t} \frac{d t}{d \theta} d \theta
$$

The transformation from time to true anomaly $(\theta)$ is approximated using the perturbation-independent term of the Gauss form of the Lagrange planetary equation for true anomaly. ${ }^{10}$

$$
\frac{d \theta}{d t} \approx \frac{h}{r^{2}}
$$

\section{G. Eclipse Arcs}

While accelerations due to solar electric propulsion only need to compute the integral in (31) through the illuminated orbital arcs, forces due to gravitational harmonics, solar radiation pressure, drag, third-body effects, and trapped radiation impingement require that the entire orbit be integrated. To determine those points at which the spacecraft enters and exits the shadow of the central body, the authors employed Escobal's quartic shadow function: ${ }^{1415}$

$$
S=\alpha_{0} \cos (\theta)^{4}+\alpha_{1} \cos (\theta)^{3}+\alpha_{2} \cos (\theta)^{2}+\alpha_{3} \cos (\theta)+\alpha_{4}
$$

where

$$
\begin{gathered}
{\left[\begin{array}{c}
\alpha_{1} \\
\alpha_{2} \\
\alpha_{3} \\
\alpha_{4} \\
\alpha_{5}
\end{array}\right]=\left[\begin{array}{ccc}
e^{4} & -2\left(\beta_{2}^{2}-\beta_{1}^{2}\right) e^{2} & \left(\beta_{2}^{2}+\beta_{1}^{2}\right)^{2} \\
4 e^{3} & -4 e\left(\beta_{2}^{2}-\beta_{1}^{2}\right) & 0 \\
6 e^{2} & -2\left(\left(\beta_{2}^{2}-\beta_{1}^{2}\right)+e^{2} *\left(1-\beta_{2}^{2}\right)\right) & 2\left(\beta_{2}^{2}-\beta_{1}^{2}\right)\left(1-\beta_{2}^{2}\right)-4\left(\beta_{1}^{2} \beta_{2}^{2}\right) \\
4 e & -4 e\left(1-\beta_{2}^{2}\right) & 0 \\
1 & -2\left(1-\beta_{2}^{2}\right) & \left(1-\beta_{2}^{2}\right)^{2}
\end{array}\right]\left[\begin{array}{c}
\alpha^{4} \\
\alpha^{2} \\
1
\end{array}\right]} \\
\alpha=\frac{r_{c b}}{p}
\end{gathered}
$$

and $\beta$ is the unitized vector from the central (shadowing) body to the Sun in the perifocal frame (PQW): ${ }^{15}$

$$
\hat{\mathbf{r}}_{s}=\beta_{1} \hat{\mathbf{p}}+\beta_{2} \hat{\mathbf{q}}+\beta_{3} \hat{\mathbf{w}}
$$


The solution of the quartic shadow funtion (33) will yield false positives, which must be filtered out. Furthermore, since we seek $\theta$ and the solution is in the form $\cos (\theta)$, an even function, we must account for all possible combinations. This yields as many as eight potential solutions for the roots, expressed in terms of $\sin (\theta)$ and $\cos (\theta)$ :

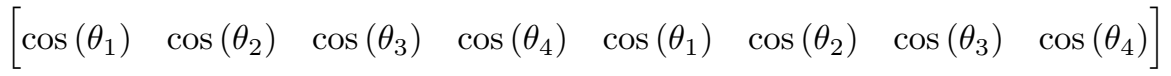

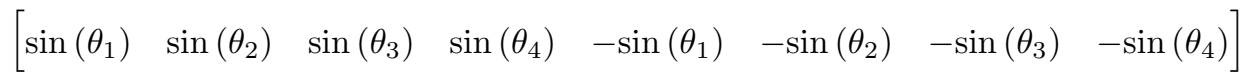

The true roots in $\cos (\theta)$ are obtained by passing (37) through the shadow function as given by Vallado (39). ${ }^{15}$ Here the shadow function has been normalized by the central body radius to help with numerical errors:

$$
S(\theta)=(1+e \cos (\theta))^{2}+\left(\frac{p^{2}}{r_{c b}^{2}}\right)\left(\beta_{1} \cos (\theta)+\beta_{2} \sin (\theta)\right)^{2}-\left(\frac{p^{2}}{r_{c b}^{2}}\right)
$$

Both Escobal's quartic shadow function (33) and the form given in Vallado (39) will also have valid roots on the illuminated side of the central body. These are filtered by assuring that we only take those roots for values of true anomaly at which central body is between the spacecraft and the Sun. Using equation 5-3 from Vallado, ${ }^{15}$ the shadow entry and exits must be such that:

$$
\beta_{1} \cos (\theta)+\beta_{2} \sin (\theta) \leq 0
$$

Now the roots of the shadow function in true anomaly are known, and the derivative of the shadow function at the roots indicates whether the root is a shadow entry $\left(\frac{d S}{d \theta}>0\right)$ or shadow exit $\left(\frac{d S}{d \theta}<0\right)$.

$$
\frac{d S}{d \theta}=2\left(\frac{p^{2}}{r_{c b}^{2}}\right)\left(\beta_{1} \cos (\theta)+\beta_{2} \sin (\theta)\right)\left(-\beta_{1} \sin (\theta)+\beta_{2} \cos (\theta)\right)-2 e \sin (\theta)(1+e \cos (\theta))
$$

We can introduce breakpoints in the integral from (31) and evaluate the integral along each illuminated or shadowed segment, applying thrust due to a solar electric propulsion system accordingly. For each interval in a given orbit, the integrand in (31) is evaluated using a ninth-order Legendre-Gauss quadrature.

\section{H. Control Parameterization}

The forces due to thrust are determined by the guidance scheme of the spacecraft. In his non-averaged scheme, Betts used a unitized thrust vector in the RSW frame as the control. ${ }^{12}$ This is not possible using an orbital-averaging approach, since a single control vector is needed that will provide the appropriate oscillatory behavior of the thrust direction within a single averaged orbit. Kluever showed that the costates of the classical orbital elements can be used as the time-varying guidance parameters for an averaging approach. ${ }^{8}$ Kluever's approach (42) assumes we only have costates governing the "slow" orbital elements, and provides a thrust vector in the velocity vector-aligned frame (NTW):

$$
a_{T} \mathbf{M}_{\mathbf{c o e}} \hat{\mathbf{u}}=\left[\begin{array}{ccc}
0 & 2 \frac{a^{2} v}{\mu} & 0 \\
\frac{r \sin (\theta)}{a \cdot v} & \frac{2(e+\cos (\theta))}{v} & 0 \\
0 & 0 & r \frac{\cos (\omega+\theta)}{h} \\
0 & 0 & r \frac{\sin (\omega+\theta)}{h \sin (i n c)} \\
-\frac{2 e+\left(r \frac{\cos (\theta)}{a}\right)}{e \cdot v} & \frac{2 \sin (\theta)}{e \cdot v} & \frac{-r \sin (\omega+\theta) \cos (i n c)}{h \sin (i n c)}
\end{array}\right]\left[\begin{array}{l}
\mathbf{a}_{n} \\
\mathbf{a}_{t} \\
\mathbf{a}_{w}
\end{array}\right]
$$


Since our equations of motion are formulated using modified equinoctial elements, the classical elements must first be computed (see reference 12). The guidance parameters are a given as a vector of the classical orbital element costates, excluding true anomaly.

$$
\lambda_{\text {coe }}=\left[\begin{array}{lllll}
\lambda_{a} & \lambda_{e} & \lambda_{i} & \lambda_{\Omega} & \lambda_{\omega}
\end{array}\right]^{T}
$$

and the thrust unit vector in the NTW frame is:

$$
\hat{\mathbf{u}}_{N T W}=\frac{-\mathbf{M}_{\mathbf{c o e}}^{\mathbf{T}} \lambda_{\text {coe }}}{\left\|\mathbf{M}_{\mathbf{c o e}}^{\mathbf{T}} \lambda_{\text {coe }}\right\|}
$$

In his work, Kluever divides $\lambda_{a}$ by the semi-major axis and parameterizes the other costates as a function of the semi-major axis. ${ }^{8}$ In this approach $\lambda_{a}$ is normalized by the semi-major axis before being used in (44), but all costates are functions of time. Notably, since the guidance parameters here are not functions of semi-major axis, the posibility exists to use these controls for a single-phase "round-trip" trajectory or other trajectories which involve both increasing and decreasing the semi-major axis of the orbit.

We may derive a similar guidance scheme where the equinoctial equations of motion and costates are used in place of the classical orbital element equations and costates.

$$
a_{T} \mathbf{M}_{\mathbf{m e e}} \hat{\mathbf{u}}=\left[\begin{array}{ccc}
0 & \frac{2 p}{w} \sqrt{\frac{p}{\mu}} & 0 \\
\sqrt{\frac{p}{\mu}} \sin (L) & \sqrt{\frac{p}{\mu}} \frac{[(w+1) \cos (L)+f]}{w} & -\sqrt{\frac{p}{\mu}} \frac{g(j \sin (L)-k \cos (L))}{w} \\
-\sqrt{\frac{p}{\mu}} \cos (L) & \sqrt{\frac{p}{\mu}} \frac{[(w+1) \sin (L)+g]}{w} & \sqrt{\frac{p}{\mu}} \frac{f(j \sin (L)-k \cos (L))}{w} \\
0 & 0 & \sqrt{\frac{p}{\mu}} \frac{s^{2}}{2 w} \cos (L) \\
0 & 0 & \sqrt{\frac{p}{\mu}} \frac{s^{2}}{2 w} \sin (L)
\end{array}\right]\left[\begin{array}{l}
\mathbf{a}_{r} \\
\mathbf{a}_{s} \\
\mathbf{a}_{w}
\end{array}\right]
$$

The guidance parameters are a given as a vector of the modified equinoctial element costates, excluding true longitude.

$$
\lambda_{\text {mee }}=\left[\begin{array}{lllll}
\lambda_{p} & \lambda_{f} & \lambda_{g} & \lambda_{j} & \lambda_{k}
\end{array}\right]^{T}
$$

and the thrust unit vector in the RSW frame is:

$$
\hat{\mathbf{u}}_{R S W}=\frac{-\mathbf{M}_{\text {mee }}^{\mathbf{T}} \lambda_{\text {mee }}}{\left\|\mathbf{M}_{\text {mee }}^{\mathbf{T}} \lambda_{\text {mee }}\right\|}
$$

In the modified equinoctial element control formulation, $\lambda_{p}$ is divided by the semi-latus rectum before being passed to (47).

The modified equinoctial element-based guidance scheme is similar to that used in SEPSPOT ${ }^{1}$ and is less prone to singularities, except when an orbit is nearly retrograde. However, if one only cares to control semi-major axis, eccentricity, and inclination of the spacecraft, this requires only three time-varying control variables $\left(\lambda_{a}, \lambda_{e}, \lambda_{i}\right)$ in the case of the classical element formulation, but five time-varying control variables $\left(\lambda_{p}, \lambda_{f}, \lambda_{g}, \lambda_{j}, \lambda_{k}\right)$ in the case of of the modified equinoctial element formulation. The additional control variables increase the size of the collocation problem, and may adversely affect runtime, though this effect has not yet been quantified.

\section{Results}

The collocation formulation of a planetocentric low-thrust trajectory optimization problem shown here is demonstrated with comparisons to the LEO to GEO and GTO to GEO cases in Kluever's demonstration of his solution method. ${ }^{8}$ 


\section{A. Minimum Time LEO to GEO}

The minimum time LEO to GEO spiral transfer uses the same assumptions as those given by Kluever. ${ }^{8}$ The spacecraft initially has a mass of $1200 \mathrm{~kg}$ and the propulsion system has a nominal power level of $10 \mathrm{~kW}$, a specific impulse of $3300 \mathrm{sec}$, and a propulsive efficiency of $65 \%$. The initial time of the propagation is January 1, 2000 and the initial state vector is given in Table 1.

\begin{tabular}{|c|c|}
\hline Variable & Initial Value \\
\hline$p$ & $6927 \mathrm{~km}$ \\
$f$ & $1.0 \mathrm{E}-6$ \\
$g$ & 0 \\
$j$ & 0.2539676 \\
$k$ & 0 \\
$m$ & $1200 \mathrm{~kg}$ \\
$r e v$ & 0 \\
\hline
\end{tabular}

Table 1. LEO to GEO initial variable values (fixed)

The solution shown here uses the classical orbital element costates as guidance parameters. The semimajor axis costate $\left(\lambda_{a} a\right)$ is fixed at -1 , giving a tangential thrust vector when $\lambda_{e}=0$ and $\lambda_{i}=0$. Costates for right ascension of ascending node $\left(\lambda_{\Omega}\right)$ and argument of periapsis $\left(\lambda_{\omega}\right)$ are fixed at 0 .

The costates corresponding to eccentricity and inclination are time-varying optimal controls. Initially the value of these controls is a linear fit of the guessed initial and final values.

\begin{tabular}{|c|c|c|c|c|c|}
\hline Parameter & Initial Value (guess) & Final Value (guess) & Lower Bound & Upper Bound & Scale Factor \\
\hline$\lambda_{e}$ & 0 & 0.5 & -1 & 1 & 100 \\
$\lambda_{i}$ & 0 & 3 & -10 & 10 & 100 \\
\hline
\end{tabular}

Table 2. LEO to GEO time varying guidance parameters

Initially, the trajectory is simulated starting at the initial state for the guessed elapsed time using linear fits to the time-varying controls. This explicit simulation provides values for the state variables at the cardinal nodes in the phase. At this point, the solution is physically accurate (the defects are approximately zero) but the constraints are not yet satisfied.

\begin{tabular}{|c|c|c|c|}
\hline Variable & Lower Bound & Upper Bound & Scale Factor \\
\hline$a$ & $42164(\mathrm{~km})$ & $42164(\mathrm{~km})$ & $\frac{1}{42164}$ \\
$e$ & 0.0001 & 0.001 & 100 \\
$i$ & $0(\mathrm{deg})$ & $0.01(\mathrm{deg})$ & 100 \\
\hline
\end{tabular}

Table 3. LEO to GEO final boundary constraints

In addition to the boundary constraints above, a path constraint is used to ensure the value of periapsis altitude is at least $300 \mathrm{~km}$. Without this path constraint, the optimizer sometimes attempts to push periapsis of the orbit below the surface of the Earth.

With the initial trajectory of the vehicle reasonably defined from the explicit simulation and the boundary and path constraints in place, the solution is solved using IPOPT. ${ }^{9}$ Reasonable convergence was achieved with the phase broken into 15 equal 3rd-order polynomial segments. The solution converged in 91 iterations. The resulting minimum transfer time is given in Table 4 including comparisons to results obtained by Kluever and SEPSPOT.

The time histories of semi-major axis, eccentricity, and inclination are shown in figure 2. They show good agreement with the results achieved by Kluever. ${ }^{8}$ Figure 3 shows the time history for the eccentricity and inclination costates used as optimal control variables. Both exhibit some "wagging" at the beginning and end of the phase. This indicates that the scaling in the collocation problem needs improvement, but the very good agreement with other results suggests that the solution is insensitive to the values of the control variables at 
those times. Better scaling may reduce or eliminate the "wagging" behavior and improve convergence but not have a significant impact on the solution.
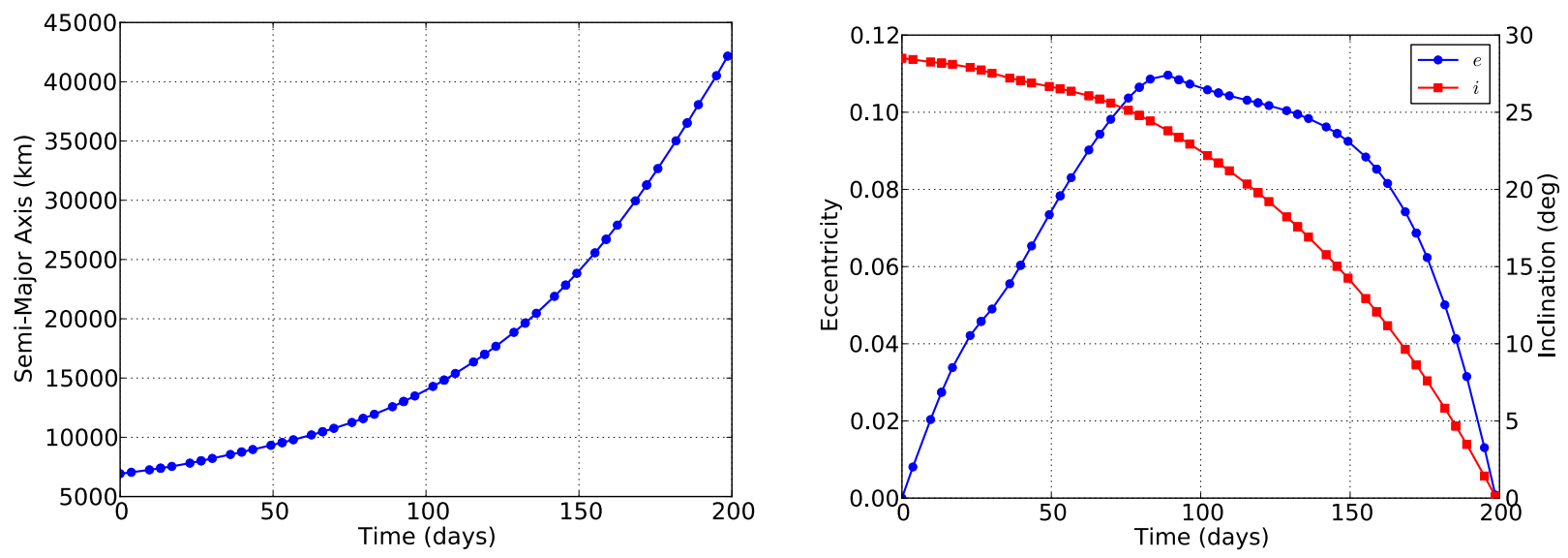

Figure 2. Semi-major axis, eccentricity, and inclination histories for the minimum-time LEO to GEO transfer. Markers indicate cardinal nodes of the collocation problem.
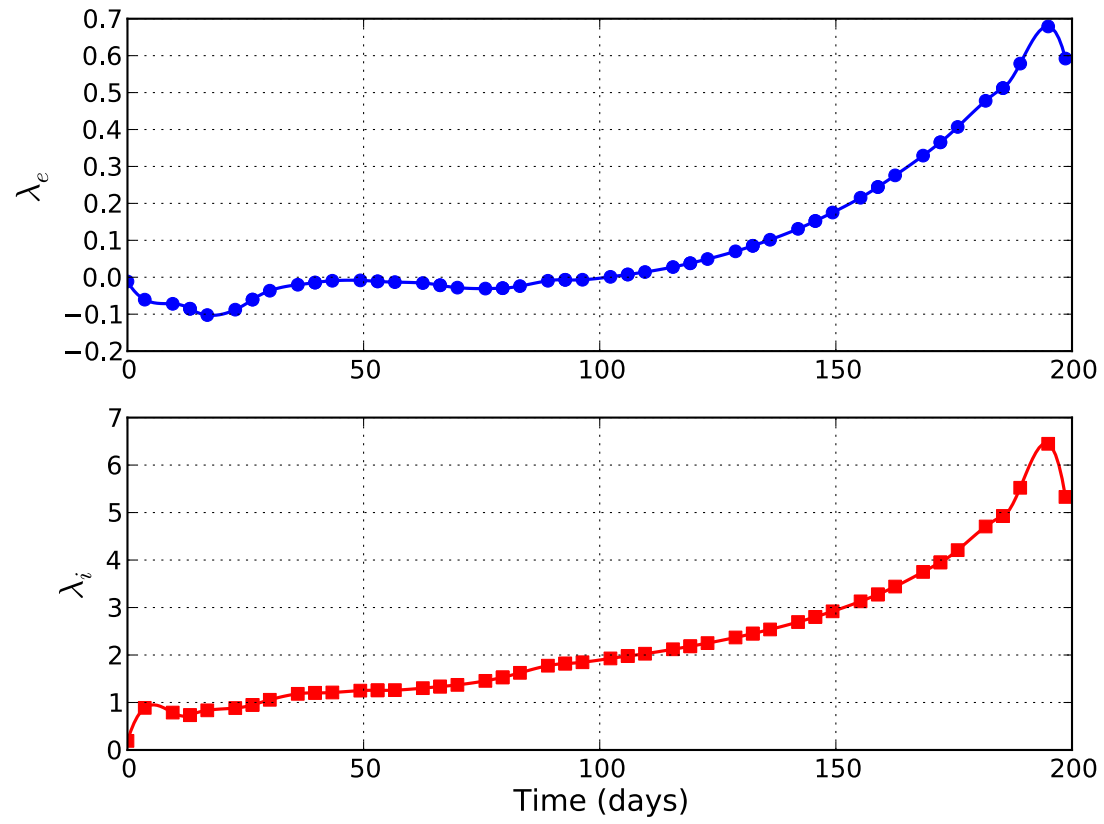

Figure 3. Eccentricity and inclination costates used as the time-varying optimal controls for the minimum-time LEO to GEO transfer.

\begin{tabular}{|l|c|}
\hline Solution Technique & Transfer Time (days) \\
\hline Collocation & $\mathbf{1 9 8 . 6}$ \\
\hline Kluever Control Parameterization $^{8}$ & 199.0 \\
\hline SEPSPOT Result $^{8}$ & 198.8 \\
\hline
\end{tabular}

Table 4. LEO to GEO results 
Figure 4 shows the evolution of the orbit from LEO to GEO for the minimum time solution, with orbits plotted at the collocation segment boundaries, roughly once every 13.2 days.
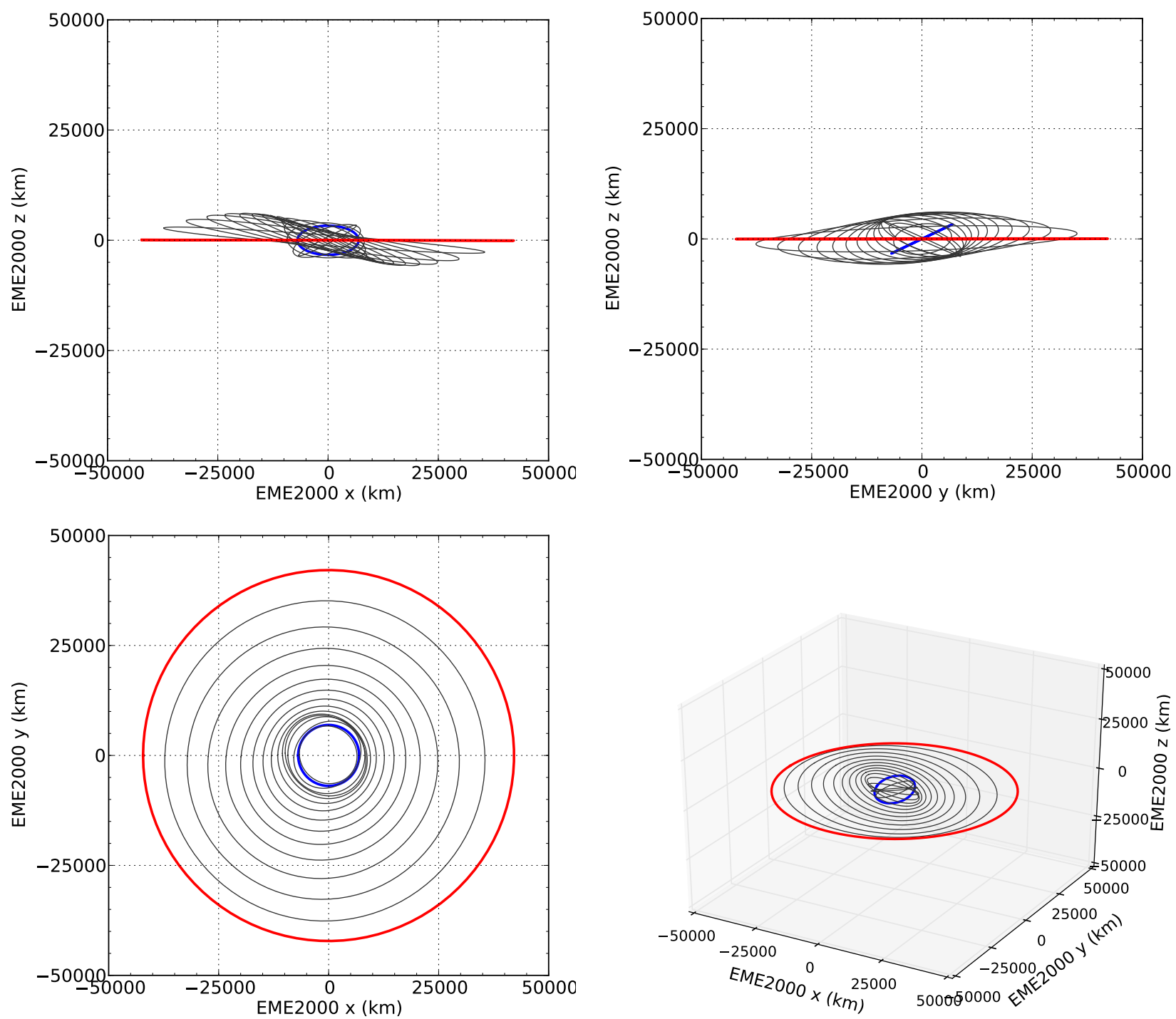

Figure 4. Evolution from the initial orbit (blue) to the final orbit (red) for the minimum time LEO to GEO transfer.

\section{B. Minimum Time GTO to GEO}

As in the previous case, the minimum time GTO to GEO spiral transfer uses the same assumptions as those given by Kluever. ${ }^{8}$ The spacecraft initially has a mass of $1200 \mathrm{~kg}$ and the propulsion system has a nominal power level of $5 \mathrm{~kW}$, a specific impulse of $1800 \mathrm{sec}$, and a propulsion system efficiency of $55 \%$. The initial time of the propagation is March 22, 2000 and the initial state vector is fixed to the values given in Table 5 .

The costates corresponding to eccentricity and inclination are time-varying optimal controls with their values linearly fit to guessed initial and final values (Table 6), and the initial state values at the cardinal nodes are obtained through an explicit simulation. The final boundary constraints are the same as those given in Table 3 and the path constraint on periapsis radius has the minimum value constrained to $185 \mathrm{~km}$.

In this case the phase was broken into 10 equal 3rd-order polynomial segments. Despite the reduction in the number of variables in the problem due to the reduced number of cardinal nodes, the GTO to GEO transfer required 299 iterations to achieve convergence. The resulting minimum transfer time is given in Table 7 with comparisons to results obtained by Kluever and results generated by SEPSPOT. 


\begin{tabular}{|c|c|}
\hline Variable & Initial Value \\
\hline$p$ & $11359.07 \mathrm{~km}$ \\
$f$ & 0.7306 \\
$g$ & 0 \\
$j$ & 0.2539676 \\
$k$ & 0 \\
$m$ & $1200 \mathrm{~kg}$ \\
$r e v$ & 0 \\
\hline
\end{tabular}

Table 5. GTO to GEO initial time and state values (fixed)

\begin{tabular}{|c|c|c|c|c|c|}
\hline Parameter & Initial Value (guess) & Final Value (guess) & Lower Bound & Upper Bound & Scale Factor \\
\hline$\lambda_{e}$ & 0.5 & 6 & 0 & 15 & 100 \\
$\lambda_{i}$ & 1 & 10 & -15 & 15 & 100 \\
\hline
\end{tabular}

Table 6. GTO to GEO time-varying guidance parameters

\begin{tabular}{|l|c|}
\hline Solution Technique & Transfer Time (days) \\
\hline Collocation & $\mathbf{1 1 8 . 2 9}$ \\
\hline Kluever Control Parameterization & 8 \\
\hline SEPSPOT & 118.36 \\
\hline
\end{tabular}

Table 7. GTO to GEO results

Figure 5 shows the time histories of semi-major axis, eccentricity, and inclination for the minimum time GTO to GEO solution, which again compare favorably with the results achieved by Kluever. ${ }^{8}$
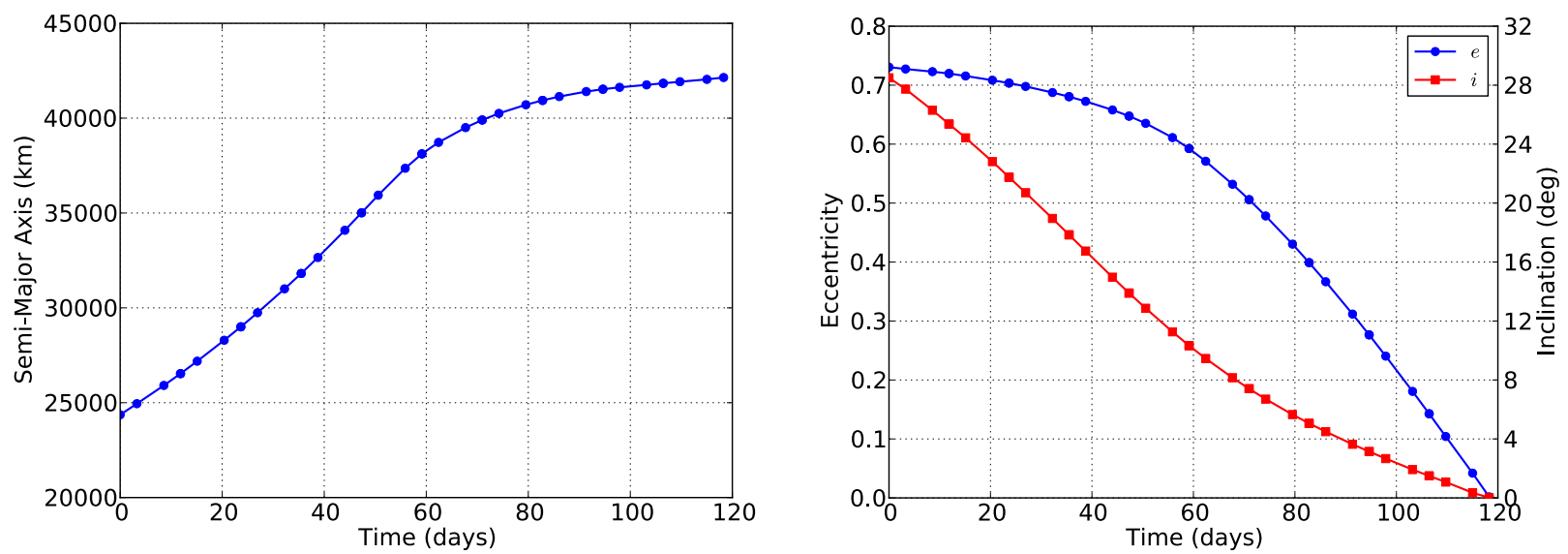

Figure 5. Semi-major axis, eccentricity, and inclination history for the minimum-time GTO to GEO transfer.

Figure 6 shows the time history for the eccentricity and inclination costates used as optimal control variables. Again, "wagging" behavior is present, especially in the inclination costate. The insensitivity of the result to the terminal value of the costates is a likely source of some of the convergence issues experienced in this case. One possible reason for this is scaling. However, the fact that the behavior seems to be exhibited where the orbit is nearly circular or near zero inclination suggest the singularities in the classical orbit element control formulation may be responsible. 

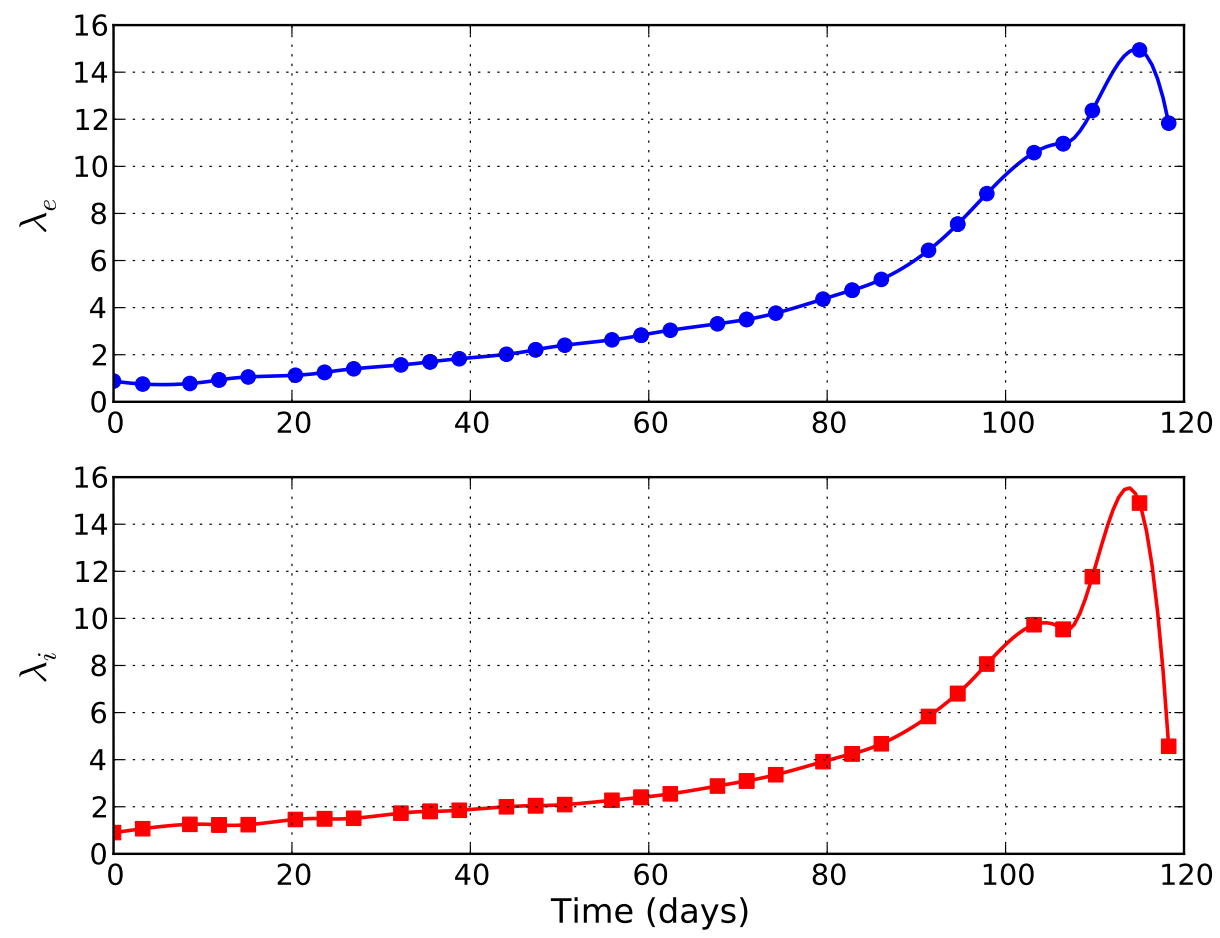

Figure 6. Eccentricity and inclination costates used as the time-varying optimal controls for the minimum-time GTO to GEO transfer.

Figures 7 and 8 show the evolution of the orbit from GTO to GEO for the minimum time solution. Orbits are plotted at the collocation segment boundaries, or approximately once every 12 days.
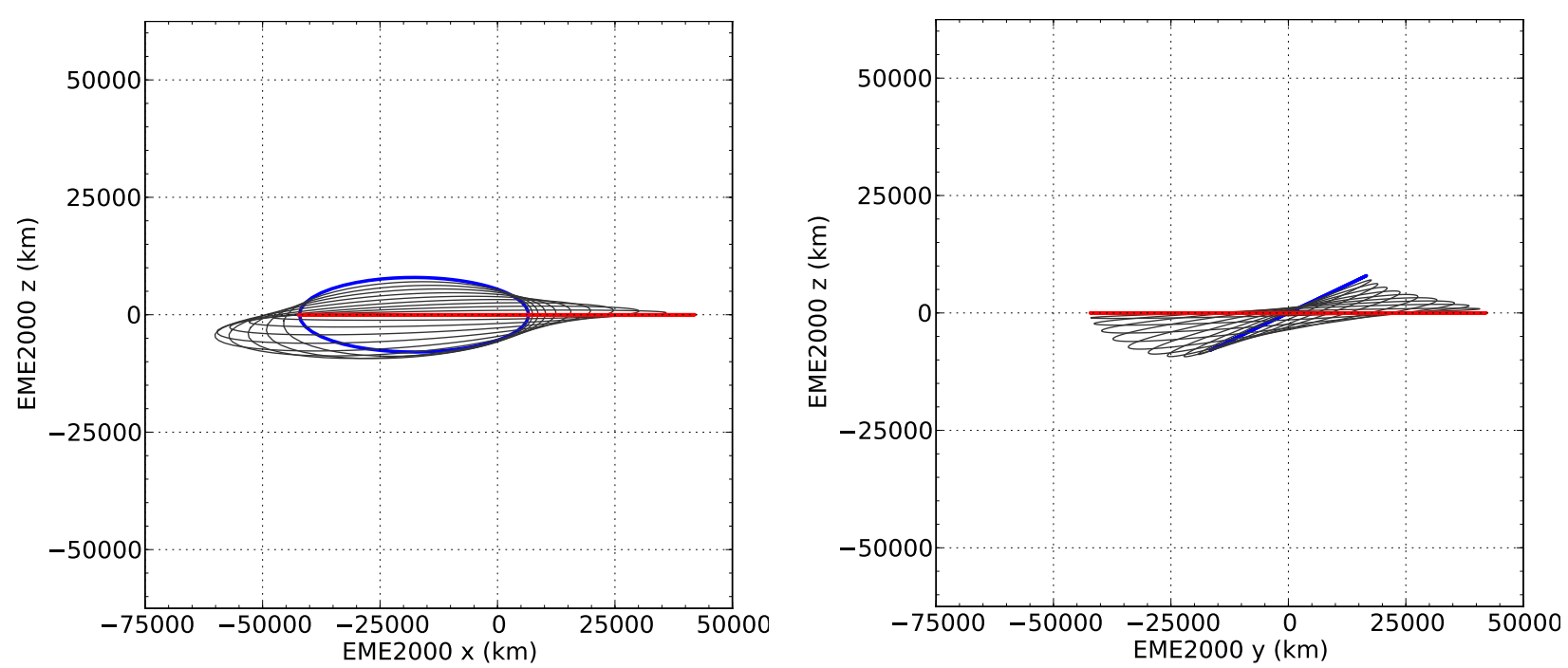

Figure 7. Evolution from the initial orbit (blue) to the final orbit (red) for the minimum time GTO to GEO transfer. 

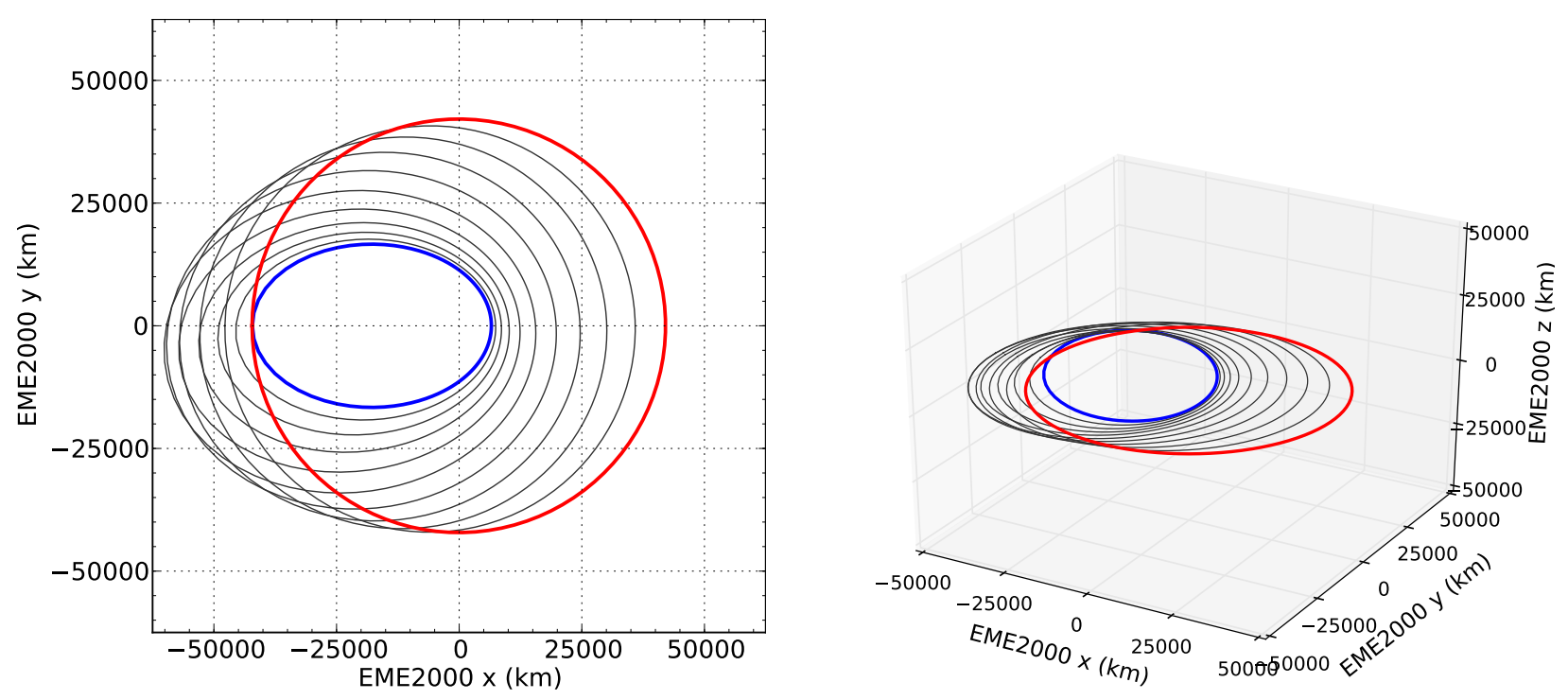

Figure 8. Evolution from the initial orbit (blue) to the final orbit (red) for the minimum time GTO to GEO transfer.

\section{Conclusions and Future Work}

The results shown here indicate that a collocation technique, combined with orbital averaging of the modified equinoctial elements, is capable of generating optimal solutions for planetocentric low-thrust orbit transfer problems relatively quickly. While the results given here were set up for comparisons with Kluever's results, ${ }^{8}$ the implementation can be extended to include other forces on the spacecraft such as atmospheric drag and perturbation by the Moon and Sun.

Despite the fact that this approach uses costates of the classical or modified equinoctial orbital elements, the use of collocation instead of a shooting method generally allows for greater convergence. Rough guesses at the guidance parameters can yield an optimal solution.

The issues of high nonlinearity in the terminal control conditions clearly need to be addressed. Better scaling of the NLP will likely help, but experience with OTIS4 has shown that other measures may be necessary. In the use of OTIS4, a common approach to such issues is to add control rate constraints, which often forces the control time history to be more smooth. A grid refinement algorithm should also be put into place to ensure that the converged solution adequately matches an explicitly integrated trajectory.

The ease of which this method can be extended to include other control formulations lends itself to experimenting with more control laws in the future. Solving these problems using the modified equinoctial element control formulations may also prove to be more robust due to the lack of singularities in those equations. Other guidance laws which do not require high-frequency time-varying controls, such as Q-Law, ${ }^{16}$ will also be explored.

Modeling the spacecraft subsystem in greater fidelity will also be a priority. Degradation of the solar arrays due to the impingement of trapped particles can be implemented as it was in SEPSPOT. Reference 1 includes a great amount of detail as to that implementation. The authors intend to implement more detailed radiation modeling in the future using more recent and higher-fidelity models.

So far this method has been demonstrated for a single phase trajectory. Using a multiple phase approach in which later phases employ non-averaged equations of motion may allow for fast, robust solutions to planetocentric spiral trajectories which target lunar orbit or lagrange points in a terminal phase. 


\section{References}

${ }^{1}$ Edelbaum, T. N., Sackett, L. L., and Malchow, H. L., "Solar Electric Geocentric Transfer With Attitude Constraints: Analysis," NASA CR 134927, National Aeronautics and Space Administration, August 1975.

${ }^{2}$ Dickmanns, E. and Well, K., "Approximate solution of optimal control problems using third order hermite polynomial functions," Optimization Techniques IFIP Technical Conference Novosibirsk, July 17, 1974, edited by G. Marchuk, Vol. 27 of Lecture Notes in Computer Science, Springer Berlin / Heidelberg, 1975, pp. 158-166.

${ }^{3}$ Hargraves, C. R. and Paris, S. W., "Direct trajectory optimization using nonlinear programming and collocation," Journal of Guidance, Control, and Dynamics, Vol. 10, Aug. 1987, pp. 338-342.

${ }^{4}$ Paris, S. W., Riehl, J. P., and Sjauw, W. K., "Enhanced Procedures for Direct Trajectory Optimization Using Nonlinear Programming and Implicit Integration," Proceedings of the AIAA/AAS Astrodynamics Specialists Conference and Exhibit, No. 2006-6309, 2006.

${ }^{5}$ Ross, I., "User's Manual for DIDO: A MATLAB Application Package for Solving Optimal control Problems," Tech. rep., TOMLAB Optimization, Sweden, 2004.

${ }^{6}$ Betts, J. T. and Huffman, W., "Sparse Optimal Control Software SOCS," Mathematics and Engineering Analysis Technical Document MEA-LR-085, The Boeing Company, Seattle, WA, 1997.

${ }^{7}$ Betts, J. T. and Erb, S. O., "Optimal Low Thrust Trajectories to the Moon," SIAM Journal on Applied Dynamical Systems, Vol. 2, January 2003, pp. 144-170.

${ }^{8}$ Conway, B. A. and Kluever, C. A., Spacecraft Trajectory Optimization, chap. 5, Cambridge University Press, New York, New York, 2010, pp. 113-137.

${ }^{9}$ Wachter, A. and Biegler, L. T., "On the implementation of an interior-point filter line-search algorithm for large-scale nonlinear programming," Mathematical Programming, Vol. 106, 2006, pp. 25-57.

${ }^{10}$ Roy, A., Orbital Motion, A. Hilger, 1988, p. 192.

${ }^{11}$ Walker, M. J. H., Ireland, B., and Owens, J., "A Set of Modified Equinoctial Elements," Celestia Mechanics, Vol. 36, July 1985, pp. 409-419.

${ }^{12}$ Betts, J., Practical Methods for Optimal Control and Estimation Using Nonlinear Programming, Advances in Design and Control, Society for Industrial and Applied Mathematics, 2010.

${ }^{13}$ Kechichian, J. A., "The treatment of the earth oblateness effect in trajectory optimization in equinoctial coordinates," Acta Astronautica, Vol. 40, No. 1, 1997, pp. $69-82$.

${ }^{14}$ Escobal, P., Methods of Orbit Determination, J. Wiley and Sons, New York, New York, 1985, pp. 155-159.

${ }^{15}$ Vallado, D. A. and McClain, W. D., Fundamentals of Astrodynamics and Applications, chap. 5, Springer, New York, New York, 2007, pp. 304-306.

${ }^{16}$ Petropoulos, A. E., "Low-Thrust Orbit Transfers Using Candidate Lyapunov Functions with a Mechanism for Coasting," Proceedings of the AIAA/AAS Astrodynamics Specialists Conference and Exhibit, No. 2004-5089, 2004. 


\begin{tabular}{|c|c|c|c|c|c|}
\hline \multicolumn{5}{|c|}{ REPORT DOCUMENTATION PAGE } & $\begin{array}{l}\text { Form Approved } \\
\text { OMB No. 0704-0188 }\end{array}$ \\
\hline \multicolumn{6}{|c|}{ 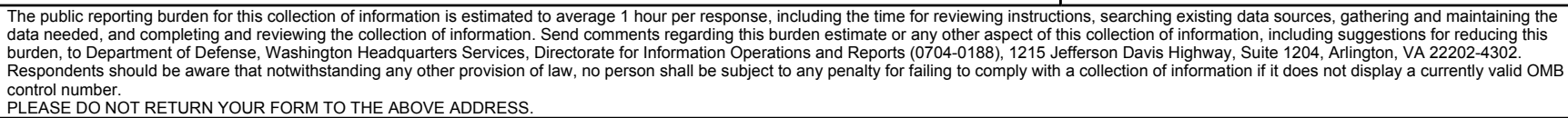 } \\
\hline \multicolumn{2}{|c|}{$\begin{array}{l}\text { 1. REPORT DATE (DD-MM-YYYY) } \\
01-09-2012\end{array}$} & \multicolumn{3}{|c|}{$\begin{array}{l}\text { 2. REPORT TYPE } \\
\text { Technical Memorandum }\end{array}$} & 3. DATES COVERED (From - To) \\
\hline \multirow{3}{*}{\multicolumn{5}{|c|}{$\begin{array}{l}\text { 4. TITLE AND SUBTITLE } \\
\text { Optimization of Low-Thrust Spiral Trajectories by Collocation }\end{array}$}} & 5a. CONTRACT NUMBER \\
\hline & & & & & 5b. GRANT NUMBER \\
\hline & & & & & 5c. PROGRAM ELEMENT NUMBER \\
\hline \multirow{3}{*}{\multicolumn{5}{|c|}{$\begin{array}{l}\text { 6. AUTHOR(S) } \\
\text { Falck, Robert, D.; Dankanich, John, W. }\end{array}$}} & 5d. PROJECT NUMBER \\
\hline & & & & & 5e. TASK NUMBER \\
\hline & & & & & $\begin{array}{l}\text { 5f. WORK UNIT NUMBER } \\
\text { WBS } 346620.02 .01 .11 .01 .01\end{array}$ \\
\hline \multicolumn{5}{|c|}{$\begin{array}{l}\text { 7. PERFORMING ORGANIZATION NAME(S) AND ADDRESS(ES) } \\
\text { National Aeronautics and Space Administration } \\
\text { John H. Glenn Research Center at Lewis Field } \\
\text { Cleveland, Ohio 44135-3191 }\end{array}$} & $\begin{array}{l}\text { 8. PERFORMING ORGANIZATION } \\
\text { REPORT NUMBER } \\
\text { E-18386 }\end{array}$ \\
\hline \multirow{2}{*}{\multicolumn{5}{|c|}{$\begin{array}{l}\text { 9. SPONSORING/MONITORING AGENCY NAME(S) AND ADDRESS(ES) } \\
\text { National Aeronautics and Space Administration } \\
\text { Washington, DC 20546-0001 }\end{array}$}} & $\begin{array}{l}\text { 10. SPONSORING/MONITOR'S } \\
\text { ACRONYM(S) } \\
\text { NASA }\end{array}$ \\
\hline & & & & & $\begin{array}{l}\text { 11. SPONSORING/MONITORING } \\
\text { REPORT NUMBER } \\
\text { NASA/TM-2012-217699 }\end{array}$ \\
\hline \multicolumn{6}{|c|}{$\begin{array}{l}\text { 12. DISTRIBUTION/AVAILABILITY STATEMENT } \\
\text { Unclassified-Unlimited } \\
\text { Subject Category: } 13 \\
\text { Available electronically at http://www.sti.nasa.gov } \\
\text { This publication is available from the NASA Center for AeroSpace Information, 443-757-5802 }\end{array}$} \\
\hline \multicolumn{6}{|c|}{ 13. SUPPLEMENTARY NOTES } \\
\hline \multicolumn{6}{|c|}{$\begin{array}{l}\text { 14. ABSTRACT } \\
\text { As NASA examines potential missions in the post space shuttle era, there has been a renewed interest in low-thrust electric propulsion for } \\
\text { both crewed and uncrewed missions. While much progress has been made in the field of software for the optimization of low-thrust } \\
\text { trajectories, many of the tools utilize higher-fidelity methods which, while excellent, result in extremely high run-times and poor } \\
\text { convergence when dealing with planetocentric spiraling trajectories deep within a gravity well. Conversely, faster tools like SEPSPOT } \\
\text { provide a reasonable solution but typically fail to account for other forces such as third-body gravitation, aerodynamic drag, solar radiation } \\
\text { pressure. SEPSPOT is further constrained by its solution method, which may require a very good guess to yield a converged optimal } \\
\text { solution. Here the authors have developed an approach using collocation intended to provide solution times comparable to those given by } \\
\text { SEPSPOT while allowing for greater robustness and extensible force models. }\end{array}$} \\
\hline \multicolumn{6}{|c|}{$\begin{array}{l}\text { 15. SUBJECT TERMS } \\
\text { Trajectory optimization; Low-thrust; Collocation; Orbital mechanics }\end{array}$} \\
\hline \multicolumn{3}{|c|}{ 16. SECURITY CLASSIFICATION OF: } & $\begin{array}{l}\text { 17. LIMITATION OF } \\
\text { ABSTRACT }\end{array}$ & $\begin{array}{l}\text { 18. NUMBER } \\
\text { OF }\end{array}$ & $\begin{array}{l}\text { 19a. NAME OF RESPONSIBLE PERSON } \\
\text { STI Help Desk (email:help@sti.nasa.gov) }\end{array}$ \\
\hline $\begin{array}{l}\text { a. REPORT } \\
\text { U }\end{array}$ & $\begin{array}{l}\text { b. ABSTRACT } \\
\text { U }\end{array}$ & $\begin{array}{l}\text { c. THIS } \\
\text { PAGE } \\
\text { U }\end{array}$ & UU & $\begin{array}{l}\text { PAGES } \\
24\end{array}$ & $\begin{array}{l}\text { 19b. TELEPHONE NUMBER (include area code) } \\
443-757-5802\end{array}$ \\
\hline
\end{tabular}



